\title{
Candidates Profile in FUVEST Exams from 2004 to 2013: Private and Public School Distribution, FUVEST Average Performance and Chemical Equilibrium Tasks Performance
}

Oliveira, R.S.A.P.; Montagna, E.; Torres, B.B.

Departamento de Bioquímica, Instituto de Química, Universidade de São Paulo (USP), São Paulo, Brazil

INTRODUCTION. Chemical equilibrium is recognized as a topic of several misconceptions. Its origins must be tracked from previous scholarship. Its impact on biochemistry learning is not fully described. A possible bulk of data is the FUVEST exam. OBJECTIVES: Identify students' errors profile on chemical equilibrium tasks using public data from FUVEST exam. MATERIAL AND METHODS: Data analysis from FUVEST were: i) Private and Public school distribution in Elementary and Middle School, and High School candidates of Pharmacy-Biochemistry course and total USP careers until the last call for enrollment (2004-2013); ii) Average performance in $1^{\text {st }}$ and $2^{\text {nd }}$ parts of FUVEST exam of Pharmacy-Biochemistry, Chemistry, Engineering, Biological Sciences, Languages and Medicine courses and total enrolled candidates until $1^{\text {st }}$ call for enrollment (20082013); iii) Performance of candidates of Pharmacy-Biochemistry, Chemistry, Engineering, Biological Sciences, Languages and Medicine courses and total USP careers in chemical equilibrium issues from $1^{\text {st }}$ part of FUVEST (2011-2013). RESULTS AND DISCUSSION: i) $66.2 \%$ of candidates came from private Elementary-Middle School courses and $71.8 \%$, came from High School courses; ii) Average grade over the period for $1^{\text {st }}$ and $2^{\text {nd }}$ FUVEST parts are respectively (in 100 points): Pharmacy-Biochemistry 66.7 and 61.2, Chemistry 65.9 and 58.9, Engineering 75.9 and 71.9, Biological Sciences 65.6 and 54.6, Languages 49.9 and 43.3, Medicine 83.5 and 79.5, total enrolled candidates 51,5 and 48.9; iii) Four chemical equilibrium issues were found during 2011-2013 and the analysis of multiplechoice percentage distribution over the courses showed that there was a similar performance of students among them, except for Engineering and Medicine with higher grades, but the same proportional distribution among choices. CONCLUSION: Approved students came majorly from private schools. There was a different average performance among courses and similar on chemical equilibrium, although better performances were in Engineering and Medicine courses and not on chemistry related.

Keywords: students school profile, chemical equilibrium, misconceptions.

Support: FAPESP (EM 2010/11608-1) 